$16^{\circ}$ Ergodesign - Congresso Internacional de Ergonomia e Usabilidade de Interfaces Humano Tecnológica: Produto, Informações Ambientes Construídos e Transporte

$16^{\circ}$ USIHC - Congresso Internacional de Ergonomia e Usabilidade de Interfaces Humano Computador

CINAHPA | 2017 - Congresso Internacional de Ambientes Hipermídia para Aprendizagem.

\title{
BOAS PRÁTICAS QUE PROPORCIONAM ACESSO À AMBIENTES DIGITAIS PARA PESSOAS COM NECESSIDADES ESPECIAIS
}

\author{
GOOD PRACTICES THAT PROVIDE ACCESS TO DIGITAL \\ ENVIRONMENTS FOR PEOPLE WITH SPECIAL NEEDS
}

\author{
Roger Morgado Curiel \\ João Carlos R. Plácido da Silva, Dr. \\ José Carlos Plácido da Silva, Dr. \\ Luis Carlos Paschoarelli, Dr. \\ (1) Universidade do Sagrado Coração \\ e-mail: roger.morgado7@gmail.com \\ (2) Universidade do Sagrado Coração \\ e-mail: joaocarlos_placido@hotmail.com \\ (3) UNESP - FAAC \\ e-mail: placido@faac.unesp.br \\ (4) UNESP - FAAC \\ e-mail: paschoarelli@faac.unesp.br
}

Acessibilidade, interface, ergonomia, inclusão, design, internet, web

No dia-a-dia a interação de pessoas com interfaces digitais está a cada vez mais presente, fazendo parte do cotidiano de diversas formas diferentes, porém nem sempre é acessível à todas as pessoas, no caso, às que possuem algum tipo de necessidade especial. Para tornar o meio digital mais democrático e incluir todos os tipos de usuários, existem diretrizes de boas práticas para os desenvolvedores de aplicações digitais seguirem.

Accessibility, interface, ergonomics, inclusion, design, internet, web

In day-to-day interaction of people with digital interfaces is increasingly present, being part of daily life in several different ways, but is not always accessible to all people, in this case, those who have some kind of special need. To make the digital environment more democratic and to include all types of users, there are good practice guidelines for digital application developers to follow. 
$16^{\circ}$ Ergodesign - Congresso Internacional de Ergonomia e Usabilidade de Interfaces Humano Tecnológica: Produto, Informações Ambientes Construídos e Transporte

$16^{\circ}$ USIHC - Congresso Internacional de Ergonomia e Usabilidade de Interfaces Humano Computador

CINAHPA | 2017 - Congresso Internacional de Ambientes Hipermídia para Aprendizagem.

\section{Introdução}

De forma geral, acessibilidade está diretamente relacionada ao compromisso de melhorar a qualidade de vida de idosos e de pessoas com deficiência (W3C; Bergman and Johnson, 1995), porém, levando em consideração o Design Universal (CORNELL et al, 1997), podemos entender acessibilidade como facilidade de aproximação e uso de ambientes e produtos abrangendo o máximo possível os grupos que podem utilizar o produto, sem discriminação.

Acessibilidade tem sido percebida como um atributo necessário à qualidade no uso de sistema de software, ou à sua usabilidade (BERGMAN AND JOHNSON, 1995; BEVAN, 1999; GRAUPP et al, 2003). Enquanto a usabilidade é definida como efetividade, eficiência e satisfação com a qual usuários específicos podem alcançar objetivos específicos em ambientes específicos (ISO, 1998), acessibilidade pode ser entendida como a flexibilidade que deve ser oferecida para interação e acesso à informação disponível, de maneira que usuários com diferentes necessidades possam acessar e usar esses sistemas (MELO E BARANAUSKAS, 2005a; W3C, 2006; CERTIC, 2006; GRAUPP et al, 2003).

Segundo Hull (2004), qualquer pessoa usando qualquer tecnologia para navegar na $\mathrm{Web}$ deveria conseguir visitar qualquer site, obter a informação que ele disponibiliza, e interagir com o site. Se um usuário não pode alcançar seus objetivos estabelecidos na interação com um sistema computacional, a usabilidade do sistema, relativa a este usuário fica comprometida (BERGMAN E JOHNSON,
1995; ISO, 1998). Um design que respeita e considera de forma indiscriminada as diferenças entre os usuários deve garantir que os objetivos estabelecidos na interação com um produto digital sejam alcançados (acessibilidade) com eficácia, eficiência e satisfação (usabilidade), na maior extensão possível (GRAUPP et al, 2003, MELO E BARANAUSKAS, 2005a).

\subsection{Como funcionam os sites acessíveis}

Conforme o portal de notícias G1 publicou em 2013, em um site acessível, conteúdo e forma andam separados. Ou seja, de um lado existe a página HTML, contendo a informação, incluindo em sua estrutura definições no código que marcam, por exemplo, o que é um título, um parágrafo, ou um link. Já a forma da página, o design propriamente dito, é definida por um arquivo chamado "folha de estilo", ou CSS (Cascading Style Sheets), como também é conhecido. Nesse documento, que é vinculado à página HTML, são definidas a apresentação visual do site, abrangendo suas cores, tamanho das letras e o espaçamento entre as linhas de texto, entre outros parâmetros.

Assim, nesse tipo de projeto, HTML e CSS são postos separados, permitindo que apenas o conteúdo textual da página seja lido por usuários que acessam por meio de ferramentas assistivas que fazem a leitura de tela. Dessa forma, informações que não interferem significativa no consumo do conteúdo para os cegos, como a cor do texto, ou o tamanho da fonte utilizada, passam a ser omitidas. Com isso, esse tipo de conceito torna o conteúdo do site adaptável a qualquer tipo de dispositivo móvel, como smartphones e tablets.

Ainda segundo o professor Edson Rufino (G1), "Quando incluo uma imagem, ou um vídeo, sem 
$16^{\circ}$ Ergodesign - Congresso Internacional de Ergonomia e Usabilidade de Interfaces Humano Tecnológica: Produto, Informações Ambientes Construídos e Transporte

$16^{\circ}$ USIHC - Congresso Internacional de Ergonomia e Usabilidade de Interfaces Humano Computador

CINAHPA | 2017 - Congresso Internacional de Ambientes Hipermídia para Aprendizagem.

um texto que tente transmitir o sentido desses elementos para os usuários que não podem acessálos, como pessoas cegas, com celulares antigos, ou com conexões mais lentas, por exemplo, estou deixando de dar a mesma oportunidade a todas as pessoas".

\section{$2 \quad$ Objetivo}

Demonstrar, na teoria, formas de boas práticas para realizar a inclusão social de pessoas com deficiência no ambiente digital com a ergonomia informacional, podendo assim proporcionar acesso a informação à este grupo de pessoas.

\section{Motivação}

Segundo o site The World Bank, um bilhão de pessoas, ou $15 \%$ da população mundial, sofrem de alguma forma de deficiência. Um quinto do total estimado global, ou entre 110 milhões e 190 milhões de pessoas, sofrem deficiências significativas.

Pessoas portadoras de algum tipo de deficiência, tendem, em média, a terem resultados socioeconômicos negativos, comparado a pessoas sem necessidades especiais, por conta da baixa nível de escolaridade, resultando também em poucas oportunidades de emprego, e até mesmo uma condição de saúde inferior.

Ainda de acordo com o The World Bank, O ambiente econômico, legislativo, físico e social de um país pode criar ou manter barreiras à participação de pessoas com deficiência na vida econômica, cívica e comunitária. As barreiras incluem edifícios inacessíveis, falta de transporte, menor acesso às tecnologias de informação e comunicação (TIC), normas inadequadas, menor nível de serviços e financiamento para esses serviços, bem como muito poucos dados e análises para sistemas baseados em evidências, políticas eficazes.

Segundo dados do Instituto Brasileiro de Geografia e Estatística (IBGE), coletados em 2010, 23,9\% dos brasileiros, ou seja, 45,6 milhões de pessoas, declararam ter algum tipo de deficiência. Dentre as apontadas, a visual foi a que mais figurou, chegando a 35,7 milhões de cidadãos. Conforme divulgado no Censo Demográfico, que pesquisou também as deficiências auditiva, mental e motora, incluindo seus graus de severidade, $18,8 \%$ das pessoas entrevistadas disseram possuir dificuldade para enxergar, até mesmo com o auxílio de óculos, ou de lentes de contato. Com relação à deficiência motora, que apareceu em segundo lugar no índice, mais de 13,2 milhões de pessoas afirmaram ter algum grau do problema, equivalente a $7 \%$ dos brasileiros.

O professor Edson Rufino (G1), que é pesquisador do Instituto Nacional da Propriedade Industrial (INPI) e membro do grupo de trabalho em acessibilidade web do $\mathrm{W} 3 \mathrm{C}$, destaca que só há vantagens em se ter um site acessível. "Em primeiro lugar, um site acessível permite o seu uso por qualquer pessoa, independente de suas características, ou formas de acesso. Em segundo lugar, projetar um site para acessibilidade normalmente gera alguns 'efeitos colaterais' bem interessantes, pois geralmente as páginas carregam mais rápido e se tornam mais fáceis de serem achadas pelos principais buscadores, como o Google, por exemplo, ficando nas melhores posições nos resultados de busca", ressalta Edson.

Desde 2004, um Decreto Federal (nº 5.296) torna obrigatório que todos os portais e sites dos órgãos da administração pública atendam aos padrões de acessibilidade digital. Depois disso, vários 
$16^{\circ}$ Ergodesign - Congresso Internacional de Ergonomia e Usabilidade de Interfaces Humano Tecnológica: Produto, Informações Ambientes Construídos e Transporte

$16^{\circ}$ USIHC - Congresso Internacional de Ergonomia e Usabilidade de Interfaces Humano Computador

CINAHPA | 2017 - Congresso Internacional de Ambientes Hipermídia para Aprendizagem.

decretos, portarias e até uma lei - a Lei de Acesso à Informação No 12.527 , de novembro de 2011 trataram do tema, abrangendo todos os sites e não apenas os governamentais. No dia 06 de julho de 2015 foi sancionada a Lei Brasileira de Inclusão da Pessoa com Deficiência (Lei 13.146) que torna obrigatória a acessibilidade nos sítios da internet mantidos por empresas com sede ou representação comercial no País ou por órgãos de governo. Mas, mesmo existindo a legislação, isso não foi suficiente para que de fato a situação tenha sido resolvida até hoje.

\section{$4 \quad$ Os padrões de acessibilidade da W3C}

A Web Accessibility Initiative da W3C publicou três guias com recomendações para tornar o ambiente web mais acessível à pessoas com necessidades especiais, assim, e não apenas criar algo exclusivo para estes.

- Guia de Acessibilidade para o Conteúdo Web (Web Content Acessibility Guidelines - WCAG) explica como tornar o conteúdo web acessível para pessoas com deficiências;

- Guia de Acessibilidade para Ferramentas de Autoria (Authoring Tool Acessibility Guidelines ATAG) - para os desenvolvedores de ferramentas que auxiliam os desenvolvedores de conteúdo a tornarem os sites acessíveis;

- Guia de Acessibilidade para Agentes do Usuário (User Agent Acessibility Guidelines - UAAG) para os desenvolvedores tornarem os documentos acessíveis aos agentes de usuários (navegadores, mídia players, ta, entre outros).

A W3C, além desses guias, disponibilizou o WCAG 2.0 (Web Content Accessibility

Guidelines), contém recomendações para a criação de conteúdo web acessível à pessoas com diversos tipos de deficiência. O documento é composto de diretrizes e princípios para os programadores e designer que criam ambientes digitais, seja online ou offline. Tais diretrizes têm a finalidade de garantir que todo o conteúdo seja compreensível e navegável à todos.

\subsection{Classificação de importância}

As regras que devem ser seguidas são divididas em três níveis de prioridade:

A - Os desenvolvedores de conteúdo digital precisam (obrigatoriamente) seguir essas instruções, caso contrário, será impossível para alguns grupos acessar o conteúdo;

AA - Os desenvolvedores deveriam (recomendado) satisfazer os requerimentos para que alguns grupos não encontrem dificuldade na utilização do produto;

AAA - Esses mesmos profissionais podem (opcional) cumprir com as diretrizes para tornar mais fácil o acesso de alguns grupos.

\subsection{Conteúdo não textual (Nível A)}

Resumo: proporcionar formas de texto para que qualquer informação que não seja textual possa ser adaptada à necessidade do usuário, como linguagens simplificadas, fala, braille e gestos.

Para esta diretriz devemos garantir que todo o conteúdo não textual também esteja disponível em texto. Considerando "texto" como texto eletrônico, e não uma imagem contendo texto como conteúdo. A vantagem deste texto é sua apresentação neutra, ou seja, pode ser processado de diversas formas como: visualmente, auditivamente, tatilmente, ou por qualquer combinação. Obtendo como resultado, as informações contidas em texto eletrônico, podem ser apresentadas da forma que melhor atenda às necessidades do usuário em questão, independente da sua necessidade. Sua fácil conversão proporciona que as informações alcancem um número maior de pessoas, adaptando-se.

Todo conteúdo não textual deve ter uma alternativa textual, como por exemplo uma imagem contendo 
$16^{\circ}$ Ergodesign - Congresso Internacional de Ergonomia e Usabilidade de Interfaces Humano Tecnológica: Produto, Informações Ambientes Construídos e Transporte

$16^{\circ}$ USIHC - Congresso Internacional de Ergonomia e Usabilidade de Interfaces Humano Computador

CINAHPA | 2017 - Congresso Internacional de Ambientes Hipermídia para Aprendizagem.

um texto descrevendo-a. Porém nem todo conteúdo não textual necessita de descrição, como é o caso de imagens decorativas, ou seja, conteúdos que não passem informações importantes.

As opções textuais são formas básicas de tornar essas informações acessíveis a todos os grupos por poderem ser processadas através de qualquer modalidade sensorial para atender às necessidades do usuário. Dar esta alternativa de texto permite que a informação seja processada de várias maneiras por uma variedade de agentes de usuário. Por exemplo, uma pessoa que não consegue ver uma imagem pode ter a alternativa de texto lida em voz alta usando discurso sintetizado. Uma pessoa que não pode ouvir um arquivo de áudio pode ter a alternativa de texto exibida para que ela possa lê10.

Essa alternativa ajuda as pessoas que têm dificuldade em perceber o conteúdo visual. A tecnologia assistiva pode ler o texto em voz alta e apresentá-lo visualmente.

Os textos também podem ajudar pessoas que têm dificuldade na interpretação de imagem, como por exemplo fotos e gráficos.

Usuários com deficiência auditiva podem ter nos textos a conversão para a linguagem gestual, como libras.

Pessoas com deficiência visual, podem ter os textos transformados em braille.

Além de auxiliar estes grupos, o conteúdo com alternativa textual, é muito bom para o mecanismo de busca (SEO, do inglês Search Engine Optimization). SEO é um conjunto de técnicas que têm como principal objetivo tornar os sites mais amigáveis para os sites de busca, trabalhando palavras-chave selecionadas no conteúdo do site de forma que este fique melhor posicionado nos resultados orgânicos.

\subsection{Mídia baseada em tempo/sincronização (Nivel A)}

Entende-se como mídia baseada em tempo/sincronização: mídias de somente vídeo, mídias apenas de áudio e mídias de áudio e vídeo.

\subsubsection{Somente áudio e somente vídeo (Pré- gravado)}

Entende-se por mídia pré-gravada aquelas que não estão sendo transmitidas em tempo real.

O objetivo desta diretriz é tornar a informação transmitida por conteúdo pré-gravado só de áudio e pré-gravado apenas de vídeo disponível para todos os usuários. As opções para mídias baseadas no tempo que são baseadas em texto tornam a informação acessível pois o texto pode ser processado através de qualquer forma sensorial (por exemplo, visual, auditiva ou tátil) para atender às necessidades dos usuários.

Esta diretriz auxilia os usuários que têm dificuldade em perceber o conteúdo visual. As tecnologias assistivas podem ajudar os usuários lendo o texto em voz alta, apresentá-lo visualmente ou até mesmo convertê-lo em linguagem de braille.

As pessoas que possuem algum tipo de deficiência auditiva ou com dificuldade de compreensão de informações de áudio por qualquer motivo, podem ler a apresentação do texto.

Usuários com deficiência auditiva e visual, podem ler às informações por braille.

\subsubsection{Mídia ao vivo}

Este critério tem o objetivo de tornar o conteúdo de vídeo e áudio transmitido em tempo real acessível a pessoas com deficiência visual e auditiva, através também da utilização de alternativa textual, mais especificamente, uma legenda sincronizada ao vivo. Neste caso será necessário um profissional para transcrever e repassar tais informações com o mínimo de atraso possível e de forma mais fiel com o acontecimento possível.

Pode ser feito de duas maneiras, com legenda em texto e com a interpretação para a linguagem de sinais (libra). 
$16^{\circ}$ Ergodesign - Congresso Internacional de Ergonomia e Usabilidade de Interfaces Humano Tecnológica: Produto, Informações Ambientes Construídos e Transporte

$16^{\circ}$ USIHC - Congresso Internacional de Ergonomia e Usabilidade de Interfaces Humano Computador

CINAHPA | 2017 - Congresso Internacional de Ambientes Hipermídia para Aprendizagem.

\subsection{Conteúdo adaptável}

A intenção desta diretriz é o desenvolvimento onde o conteúdo que possa ser mostrado de diferentes formas, entretanto, sem perder as informações e mantendo a estrutura organizacional, como por exemplo um layout mais simples e intuitivo onde possa se tornar mais acessível a todos os tipos de usuários.

\subsection{Diferenciais (nível A)}

Diferentemente das diretrizes anteriores, esta tem como objetivo estabelecer um padrão para o conteúdo, tornando-o mais intuitivo e contrastante.

São exemplos de medidas para serem tomadas:

- Contraste de cores;

- Contraste do primeiro plano em relação ao segundo, seja contraste visual ou auditivo;

- Utilização de fontes legíveis;

- Diferenciação de elementos que clicáveis (como textos linkados e botões);

- Diferenciação de elementos selecionados, habilitado e desabilitado;

- Diferenciação em relação ao nível do título, como por exemplo título e subtítulo.

\subsection{Operacional (nível A)}

Disponibilizar todas as funcionalidades a partir de um teclado virtual. Toda ação pode ser executada utilizando este teclado, com reconhecimento de voz, pelo mouse e por outras diversas tecnologias assistivas.

\subsection{Tempo ajustável}

Esta diretriz diz respeito a possibilitar aos usuários tempo suficiente para consumir o conteúdo exposto e concluir ações, o ideal é que eles mesmos possam escolher o seu próprio tempo. Esta autonomia é importante por conta da reação física ser mais dificultosa, consequentemente mais lenta, como a dificuldade de leitura, e até mesmo podem estar acessando o conteúdo por uma tecnologia assistiva que necessita de mais tempo.

\subsection{Navegável (nível A)}

O objetivo desta diretriz é auxiliar os usuários a encontrar a informação que estão buscando. Usuários digitais que possuem necessidades especiais encontram mais dificuldades em realizar essa tarefa, por isso é importante sempre informar de forma explícita e linearmente: de onde vim, onde estou e pra onde posso ir.

É importante também definir uma order linear de foco na sequência correta do conteúdo, e também mostrar onde está o foco por conta de usuários que navegam utilizando o teclado, vão navegando pela página sequencialmente onde o foco as levam. Tal ordem é definida pelo código HTML.

Oferecer mais de uma alternativa para os usuários encontrar a informação que procura é recomendado (nível AA), pois determinado usuário pode achar a forma $\mathrm{B}$ mais fácil que a forma $\mathrm{A}$.

As páginas e todo o conteúdo deve-se também ser devidamente intitulado para que as informações sejam localizadas com mais facilidade (nível AAA).

\subsection{Legibilidade (nível A)}

O foco desta diretriz é quanto a legibilidade do texto, permitindo que o conteúdo textual possa ser lido não apenas pelos usuários, mas também pelas tecnologia assistiva.

A diversas maneiras deste tipo de conteúdo ser passado para os usuários, com experiência diversificadas, com a leitura visual, para outros o consumo do conteúdo pode ser feito pela audição e até mesmo de forma tátil como é o caso do braille.

\subsubsection{Idioma da página}

Identificar o idioma da página auxilia as ferramentas assistivas a repassar o conteúdo corretamente para o usuário, como a pronúncia.

\subsubsection{Mecanismo de Pronúncia (nível AAA)}

Um simples acento pode mudar a pronúncia que 
$16^{\circ}$ Ergodesign - Congresso Internacional de Ergonomia e Usabilidade de Interfaces Humano Tecnológica: Produto, Informações Ambientes Construídos e Transporte

$16^{\circ}$ USIHC - Congresso Internacional de Ergonomia e Usabilidade de Interfaces Humano Computador

CINAHPA | 2017 - Congresso Internacional de Ambientes Hipermídia para Aprendizagem.

consequentemente pode mudar completamente o sentido do texto, dificultando assim a compreensão de pessoas com deficiência visual ou que tenha alguma dificuldade de leitura. As ferramentas assistiva como leitores de tela podem falar os caracteres incorretamente sem as informações sobre a pronúncia, por isso é importante disponibilizar alternativas para solucionar este problema, como um arquivo de som da pronúncia e um glossário.

\subsection{Assistência}

Usuários portadores de algum tipo de deficiência, tendem a cometerem mais erros e ainda há uma certa dificuldade em perceber o erro que cometeu. O objetivo desta diretriz é reduzir estes erros e que os erros cometidos, sejam percebidos com maior facilidade. Fornecer informações sobre erros de entrada no texto permite que os usuários cegos ou daltônicos percebam o fato de que ocorreu um erro.

Quando um for cometido, deve-se (nível A) avisar com uma mensagem de erro especificando o campo e o erro que foi cometido para o usuário ter conhecimento do ocorrido e para que possa corrigir sem maiores dificuldades. A falta deste recurso pode acarretar no abandono por parte do utilizador.

Para facilitar o preenchimento de informações como em um formulário, recomenda-se (nível A) colocar instruções claras de como fazer a utilização corretamente daquele conteúdo.

Se um erro na inserção de informações for cometido, é altamente recomendado (nível AA) as sugestões de correções, com algumas exceções como em casos que comprometa a finalidade do conteúdo ou até mesmo a segurança.

Tratando-se de conteúdo que causem transações financeiras ou compromissos legais, e que exclua e modifique informações que o usuário tem controle, deve-se ter pelo meno umas das seguintes instruções para evitar consequências graves por conta de um erro causado muitas vezes pela sua limitação:

- Reversível: As inscrições são reversíveis.

- Confirmado: As informações fornecidas pelo usuário devem ser verificadas, e caso haja erro de inserção, deve-se proporcionar a oportunidade de correção.

\section{$5 \quad$ Benefícios da Acessibilidade na Web}

Os itens abaixo em checklist são argumentos das vantagens em seguir às boas práticas.

- Maior valor agregado à empresa e fortalecimento da sua marca;

- Melhoria de performance e de usabilidade, criando vantagem competitiva;

- Ampliação e diversificação de público;

- Fidelização de usuários e clientes;

- Maior visibilidade em buscadores como Google, Bing etc.;

- Facilidade e menor custo na manutenção de conteúdo;

- Aumento de compatibilidade com todos os dispositivos, plataformas, sistemas e navegadores.

\section{$6 \quad$ Ferramentas que auxiliam a construção de websites acessíveis}

Os itens abaixo em checklist são recursos já existentes que ajudam no desenvolvimento.

- Check my Colours: ferramenta utilizada para testar o contraste textual de seu website.

- Contrast Ratio: conferir o contraste entre o background e a cor do texto.

- ProDeaf - WebLibras: ferramenta que permite implementar um tradutor de libras para os textos do site. 
$16^{\circ}$ Ergodesign - Congresso Internacional de Ergonomia e Usabilidade de Interfaces Humano Tecnológica: Produto, Informações Ambientes Construídos e Transporte

$16^{\circ}$ USIHC - Congresso Internacional de Ergonomia e Usabilidade de Interfaces Humano Computador

CINAHPA | 2017 - Congresso Internacional de Ambientes Hipermídia para Aprendizagem.

- Wave Evaluation Tool: Extensão do Chrome para teste de acessibilidade em websites.

\subsection{Navegadores textuais e leitores de tela}

Opções de navegadores para ver na prática como é o funcionamento de ferramentas e se aproximar ainda mais dos usuários finais

\footnotetext{
- Dosvox;

- Jaws;

- Lynx
}

\section{$7 \quad$ Testes para checar a acessibilidade do site}

Existem alguns testes básicos que podem ser realizados com a finalidade de verificar os itens mais importante da acessibilidade no site. A listagem a seguir mostra procedimentos que se constituem em um bom começo para incrementar a acessibilidade do site para pessoas portadoras de necessidades especiais:

\subsection{Textos alternativos para imagens que transmitam informação}

Posicione o cursor sobre a imagem, deverá aparecer um tipo caixinha com um texto descritivo da imagem. Para usuários navegando com browsers sem suporte para imagens, será aquele texto descrito, visto (e/ou ouvido) no lugar da imagem.

\subsection{Textos alternativos para imagens \\ decorativas}

Neste caso, essa caixinha descrevendo a imagem é desnecessária por se tratar apenas de uma imagem decorativa que não passa informação relevante.

\subsection{Consuma conteúdo de vídeo e áudio sem volume}

Tirar totalmente o som, simula na prática usuários portadores de deficiência auditiva. Caso não tenha sido possível entender o conteúdo destas mídias, é necessário disponibilizar o mesmo conteúdo de forma escrita.

\subsection{Acessibilidade dos formulários}

Geralmente cada campo de um formulário possui seu texto indicando qual informação deve ser inserida naquela área. Clique sobre o texto descritivo do campo e deverá aparecer o cursor 'piscando' no campo que clicou, se o cursor não aparecer é porque não foi usado o elemento ' $<$ label $>$ ' no formulário e isto poderá tornar o formulário inacessível para alguns usuários.

\subsection{Opção de aumentar o tamanho das letras}

Acesse as configurações do navegador que está utilizando e aumente o tamanho da fonte, caso não tenha alterado, significa que o site testado estará inacessível para usuários portadores de baixa visão.

\subsection{Testar o site com o navegador Lynz}

O browser Lynx é um browser de texto bem simples comparado aos outros browsers, com ele é possível conferir como o site irá se comportar com ferramentas assistivas que fazem uma leitura do site.

\subsection{Navegabilidade sem uso de um mouse}

Tente navegar pelo site utilizando apenas o teclado, utilize $t a b$, shift + tab e return. Caso não obtenha sucesso, significa que o site é inacessível para usuários impossibilitados de usar mouse, assim como à usuários com leitores de tela.

\subsection{Mapa do site}

Consegue localizar facilmente o mapa do site? Caso a resposta seja negativa, há grandes chances de alguns usuários ficarem perdidos no site e desistirem de utilizá-lo.

\subsection{Textos dos links}

O texto do link deve descrevem qual será o destino caso o usuário clique nele. 'Clique aqui' e 'mais' são 
$16^{\circ}$ USIHC - Congresso Internacional de Ergonomia e Usabilidade de Interfaces Humano Computador

CINAHPA | 2017 - Congresso Internacional de Ambientes Hipermídia para Aprendizagem.

dois exemplos bem comuns de textos não descritivos para links. Caso não seja possível colocar uma descrição no link, deve-se utilizar a tag title do HTML no elemento $a$ para suprir a ausência da descrição.

\section{$8 \quad$ Conclusão}

Está pesquisa apresentou diversas práticas ergonomicas que possibilita a inclusão de um número maior de pessoas.

Ao desenvolver um produto digital, programadores, designers e todos os inúmeros profissionais ligados a este setor, deve ser sensível às diferenças existentes entre todas as personas $\mathrm{e}$ oferecer um ambiente flexível que possibilite que cada pessoa participe sem discriminação.

Apesar das inúmeras diretrizes existentes, quando não é feito um sólido planejamento prévio, há algumas dificuldades no desenvolvimento, considerando requisitos de acessibilidade, $o$ principal fator que pesa contra é o tempo, é necessário ter um tempo maior na produção da aplicação e mão de obra especializada, o que resulta em um custo maior de produção, desencorajando empresas a considerar essas boas práticas.

De acordo com o professor Edson Rufino, tornar um site acessível não onera um projeto de site. "Como qualquer melhoria que tentamos implementar, a acessibilidade só se torna caro se tentamos fazer adaptações a algum projeto que já esteja pronto, ou em estágio avançado de desenvolvimento. $\mathrm{O}$ ideal é projetar um site desde o início com o foco na acessibilidade. Neste caso, não há diferença significativa no custo de desenvolvimento". 
$16^{\circ}$ Ergodesign - Congresso Internacional de Ergonomia e Usabilidade de Interfaces Humano Tecnológica: Produto, Informações Ambientes Construídos e Transporte

$16^{\circ}$ USIHC - Congresso Internacional de Ergonomia e Usabilidade de Interfaces Humano Computador

CINAHPA | 2017 - Congresso Internacional de Ambientes Hipermídia para Aprendizagem.

\section{Referências}

Bergman, E., Johnson, E. (1995). “Towards Acessible Human-Computer Interaction". In: Nielsen, J. (ed), Advances in Human-Computer Interaction, Ablex Publishing.

Bevan, N. (2001). "Quality in use for all". In: Stephanidis, C. (ed.), User Interfaces for All: Concepts, Methods, and Tools, Lawrence Erlbaum.

Certic (2006). Acessibilidade à Web, n.d. Acesso: Março de 2017, site Centro de Engenharia de Reabilitação em Tecnologias de Informação e Comunicação: http://www.acessibilidade.net/web/

Connell, B. R., Jones, M., Mace, R. Et al (1997). "The Principles of Universal Design", Version 2.0. Raleigh.

G1. Acesso: 24 de março de 2017, site:

http://redeglobo.globo.com/globociencia/noticia/20 13/06/sites-acessiveis-permitem-que-pessoas-comdeficiencia-usem-web.html

Google. Acesso: 24 de março de 2017, site: https://developer.android.com/guide/topics/ui/acces sibility/index.html

Graupp, H., Gladstone, K., Rundle, C. (2003). "Accessibility, Usability and Cognitive Considerations in Evaluating System with Users who are Blind". In: Stephanidis, C. (ed), Universal Access in HCI: Inclusive Design in Information Society, Vol. 4, Crete, 22-27, Lawrence Erlbaum Associeates, pp. 1280-1284.

ISO (1998). “ISO 9294-11 Ergonomic requirements for office work with visual display terminals - Part 11", Guide on usability.

Maujor - O Dinossauro das CSS. Acesso 24 de março de 2017, site: www.maujor.com/tutorial/acessibilidade/tentest.ph $\mathrm{p}$

Melo, A. M., Baranauskas, M. C. C. (2005a). Design e Avaliação de Tecnologia Web-acessível”.
In: Jornadas de Atualização em Informática, XXV Congresso da Sociedade Brasileira de Computação, São Leopoldo - RS, pp. 1500-1544.

Melo, A. M., Baranauskas, M. C. C. (2005b). "Ambientes Virtuais Inclusivos: Desafios ao Design para Acessibilidade". In: The Latin American Conference on Human-Computer Interaction, Cuernava - México, pp. 339-339.

The World Bank. Acesso: 24 de março de 2017, site:

http://www.worldbank.org/en/topic/disability/ overview\#1

W3C (2008). "Web Acessibility Initiative". Acesso: Fevereiro de 2017, site do World Wide Web Consortium: www.w3.org/WAI
Realização:

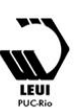

